\title{
Percutaneous Reduction and Fixation of Displaced Intra-Articular Calcaneal Fractures
} A.S.Allam, A.A.Ahmad, H.A.El-Attar and M.A.Al-Mansy

Orthopaedic Surgery Dept., Faculty of Medicine, Benha Univ., Benha, Egypt

E-Mail: malek.ahmad381988@gmail.com

\begin{abstract}
Despite the relative prevalence of intra-articular calcaneal fractures, definitive management is controversial. The goal of this study is to evaluate the clinical and radiological outcome of percutaneous reduction and fixation of 20 patients with intraarticular calcaneal fractures Sanders type II, III or IV by k wires, Steinmann pins or cannulated screws subjectively and objectively over 8 months. Twenty-two (22) percutaneous fixation of calcaneal fractures in 20 patients were included in this study. This study included closed intra-articular calcaneal fractures type II , III and IV in adult patients and excluded open, extra-articular, type I fractures. The American Orthopaedic Foot and Ankle Score (AOFAS) was used for evaluation. The excellent and good results were considered satisfactory while the poor and fair results were considered unsatisfactory. The results at the end of this study were satisfactory in $86.3 \%$ of patients (AOFAS score more than 75 in 19 fractures) and unsatisfactory in $13.6 \%$ of patients (AOFAS score less than 75 in 3 fractures). All patients included in this study showed normal range of motion of the ankle joint (active dorsiflexion and planter flexion) with variable decrease in the subtalar range of motion (active inversion and eversion) when compared with the normal side. .Percutaneous reduction and fixation had been found to be satisfactory in reduction of Böhler's angle, Gissane angle and Sarrafian angle with avoiding open reduction surgical and postoprtative complications and avoiding conservative management bad functional outcome.
\end{abstract}

Keywords: Intra-articular, Calcaneal fractures, percutaneous reduction, fixation.

\section{Introduction}

The calcaneal bony anatomy is complex as it is irregularly cuboid in shape and its long axis is directed forwards, upwards, and somewhat laterally. There are two articulations (with three facets) with the talus and one saddle-shaped articulation with the cuboid [1].

Calcaneal fractures have long been recognized as a source of significant disability and remain one of the most difficult articular fractures to treat [2].

Displaced intraarticular fractures of the calcaneus generally occur in the event of high energy trauma. The pattern of fracture lines and extent of comminution depend on the position of the foot at impact, the extent of force involved at impact, and the patient's overall bone quality. The initial radiographic evaluation includes a lateral view of the hindfoot, an anteroposterior (AP) view of the foot, a Harris axial view of the heel, Broaden view of subtalar joint and a mortise view of the ankle. Plain radiographs of the lumbar spine are also routinely obtained [3].

CT scanning has truly revolutionized our understanding of the pathoanatomy of calcaneal fractures, images are obtained in 2-3 $\mathrm{mm}$ intervals in the axial, sagittal, and 30-degree semicoronal planes [4].

Classification systems based on plain radiography have been only marginally useful, in part because of limited accuracy in delineating fracture patterns. The development of CT leads to the development of classification systems. The Sanders classification system for intraarticular fractures is the most commonly used [5].

Treatment of intraarticular calcaneal fractures can be divided into conservative and operative management. The operative management includes open reduction and internal fixation, percutaneous reduction and fixation and primary arthrodesis. Conservative treatment might be considered in non-displaced or minimally displaced fractures, compromised soft-tissues and in patients with physical contra-indications (e.g., peripheral vascular disease, psychic patient).Open reduction and internal fixation is considered the gold standard treatment for displaced intraarticular fractures of the calcaneus by most surgeons as it generally provides overall good to excellent results and the ability to anatomically restore the subtalar joint. Disadvantages of open surgical approaches are wound dehiscence and infection which may occur in up to $30 \%$ of patients $[6,7,8]$.

The goal of this study is to evaluate the clinical and radiological outcome of percutaneous reduction and fixation of 20 patients with intraarticular calcaneal fractures Sanders type II, III or IV by k wires, Steinmann pins or cannulated screws subjectively and objectively over 8 months using The American Orthopaedic Foot and Ankle Score (AOFAS).

\section{Patient and method}

Twenty-two percutaneous fixation of calcaneal fractures in 20 patients were included in this study. This study was done at Matarya teaching hospital and Benha university hospitals between July 2019 and January 2020.These patients were followed up for at least 32 weeks post-operatively.

\section{Patient's inclusion criteria}

- Fracture type: Displaced Intra-Articular Sander type II, III and IV.

- Patient's age: skeletally mature.

- Unilateral or bilateral Fracture.

\section{Patients Exclusion criteria:}

- Open fractures.

- Ostoporotic fractures.

physical contra-indications (e.g., peripheral vascular disease, psychic patient, poor general condition, paralysed limb). 
The postoperative protocol employed an immediate postoperative radiograph (lateral and axial views) and measurement of the three angles: Gissane, Bohler's angle and posterior facet inclination angles.

The patients started postoperative ankle mobilization with non-weight bearing axillary crutch mobilization on postoperative day three.

Follow up at outpatient clinic for 32 weeks at 1 st week, 2nd weeks, 1st month, 2nd month, 4th month, 6th month and 8th month.

Partial weight bearing mobilization was begun at six weeks, full weight bearing at 12 weeks. At 8th months the final review was made, and ankle function was scored as per The American Orthopaedic Foot and Ankle Score (AOFAS) system.

The complications were documented, The patients were evaluated by The American Orthopaedic Foot and Ankle Score (AOFAS) system.

It is a score of 100 points. The score is strictly clinical with no radiologic factor included. Both subjective and objective clinical factors constituted the scale. A score of 90-100 is considered excellent, 75-89 is considered good, 50-75 is considered fair, and less than 50 is considered poor Table (1) [2].

Anteroposterior, lateral and Harris axial views were obtained for all patients immediately after surgery. Xrays were done for all patients at 1 st week, 2nd weeks, 1st month, 2nd month, 4th month, 6th month and 8th month. Additional X-rays were also done at any time following the operation if the patient had pain. Postoperative CT ankle were done if possible. Loss of reduction was defined as the appearance of $\geq 2 \mathrm{~mm}$ of displacement of the joint or the body of the calcaneus.

\section{Results}

The maximum age incidence in this study was from $30-40(45 \%)$ and the least age incidence was from 40-70 $(25 \%)$. The mean age was $36.45 \pm 11.213$ (range from 19 to 59) years old.18 patients $(90 \%)$ were males and 2 females $(10 \%)$ showing male predominance. $\mathrm{M}: \mathrm{F}=9: 1$.

13 patients $(65 \%)$ were broken on the right side and $5(25 \%)$ patients on the left side. There were 2 patients $(10 \%)$ of bilateral involvement.Only 3 patients had associated injuries which included: fracture tibial shaft in 1 patient, lumbar spine fracture in 1 patient and fracture femur in 1 patient.

The predominant mechanism of injury was fall from height and landing on the heel in 18 patients (90\%). The other mechanism was road traffic accident in 2 patients (10\%).All fractures were classified using Sander's Classification for fracture of calcaneus. 15 (68.2\%) calcaneal fractures were Type II, $5(22.7 \%)$ calcaneal fractures were Type III and 2 (9.09\%) calcaneal fractures were type IV.

In this study, According to AOFAS scores, the excellent and good results were considered satisfactory while the poor and fair results were considered unsatisfactory. The results at the end of this study were satisfactory in $86.3 \%$ of patients (AOFAS score more than 75 in 19 fractures) and unsatisfactory in $13.6 \%$ of patients (AOFAS score less than 75 in 3 fractures).

All patients included in this study showed normal range of motion of the ankle joint (active dorsiflexion and planter flexion) with variable decrease in the subtalar range of motion (active inversion and eversion) when compared with the normal side. The subtalar joint was examined by holding the calcaneus with one hand and the talar head with thumb and index fingers of the other hand and applying varus and valgus stress. The average subtalar range of motion at follow up was equivalent to about $80 \%$ of the contralateral healthy foot.The mean time of complete radiological union was 12 weeks.

The mean pre-operative Böhler's angle was $14.08 \pm 2.439^{\circ}$ (range $9-17^{0}$ ) and the mean postoperative Böhler's angle was $26.15 \pm 4.187$ (range 18 $33^{0}$ ). The result was found to be highly significant ( $\mathrm{p}$ value $=<0.001$ ) using paired-samples $\mathrm{T}$ test. This means improvement of Böhler's angle after fixation achieved in 14 fractures $(63.6 \%)$ and not achieved in 8 fractures (36.4\%).Restoration of normal range Bohler angle (2540) was achieved in 14 fractures $(63.6 \%)$ and not achieved in 8 fractures $(36.4 \%)$.

The mean pre-operative Gissane angle was $151.14 \pm 14.264^{\circ}$ and the mean post-operative Gissane angle was $136.32 \pm 15.255^{\circ}$. The result was found to be highly significant using paired-samples $\mathrm{T}$ test ( $\mathrm{p}$-value = $<0.001)$. This means improvement of Gissane angle after fixation in 12 patients $(52.2 \%)$ and not achieved in 10 patients $(47.8 \%)$. Restoration of normal range Gissane angle $\left(120^{\circ}-145^{\circ}\right)$ was achieved in 12 patients $(52.2 \%)$ and not achieved in 10 patients $(47.8 \%)$. The mean preoperative Sarrafian angle was $40.50 \pm 7.769$ and the mean post-operative Sarrafian angle was $55.32 \pm 8.555$. The result was found to be highly significant using paired-samples $\mathrm{T}$ test $(\mathrm{P}$-value $<0.001)$. This means improvement of Sarrafian angle after fixation in 9 patients $(43.5 \%)$ and not achieved in 13 patients $(56.5 \%)$

Restoration of normal range Sarrafian angle $\left(55^{\circ}-75^{\circ}\right)$ was achieved in 9 patients $(43.5 \%)$ and not achieved in 13 patients $(56.5 \%)$

According to age distribution, Restoration of normal angles post-operatively was achieved in 5 fractures for one angle, in 11 patients for two angles and in 6 fractures for the three angles. The result was statistically insignificant when comparing age distribution in the three groups using one-way analysis of variance test ( $\mathrm{p}$ value: 0.388). According to gender distribution, Restoration of normal angles post-operative was achieved in 4 males and one female for one angle, in 9 male patients and 2 females for two angles and in 6 male patients for the three angles. The result was statistically insignificant using Fisher's exact test ( $p$ value: 0.571$)$.

According to mechanism of injury, Restoration of normal angles post-operatively was achieved in 5 patients (4 patients due to fall from height and one patient due to road traffic accident) for one angle, two angles were achieved in 9 patients due to fall from height and two patients due to road traffic accident and the three 
angles were achieved in 5 patients due to fall from height. The result was statistically insignificant using Fisher's exact test (p-value: 0.571).

According to side of injury Restoration of normal angles post-operative was achieved in one angle in 5 patients ( 1 right, 2 left and 2 bilateral), in two angles in 6 right side, 3 in left and 2 bilateral and in three angles in 6 right-sided fractures. The result was statistically insignificant using Fisher's exact test (p value: 0.093).

According to fracture classification, Restoration of normal angles post-operative was achieved in one angle in 5 patients ( 3 type III, 2 type IV), in two angles in 11 patients ( 9 type II and 2 type III) and in three angles in 6 patients all type II. The result was statistically significant using Fisher's exact test ( $p$ value: 0.001 ).

According to duration from injury to surgery, Restoration of normal three angles post-operative was achieved in 6 patients done early than others by 0.43 day. That result was statistically insignificant using one-way analysis of variance test ( $\mathrm{p}$ value was 0.289 ).

Extra screw was used for fixation of other fragments in 5 fractures (22.7\%).Eighteen patients (90\%) started weight bearing at 12 weeks while two patients (10\%) started weight bearing at 16 weeks. The mean age of the satisfactory results was $36.47 \pm 12.743$ years. The mean age of the fair results was $38.00 \pm 5.000$ years. This was found to be statistically insignificant. (pvalue:0.523) .

The results were found to be statistically nonsignificant (p-value: 0.459). Fisher's exact test was used.This was found to be statistically non-significant ( $p$ value: 0.456 ). Fisher's exact test was used.

$71.4 \%$ of the excellent results were obtained in sanders type II fractures, while $28.6 \%$ of the excellent results were obtained in sanders type III fractures. This was found to be statistically significant (p-value: 0.019).
This indicates that outcome correlate with severity of fracture.

The mean time from injury to operation in satisfactory results was $4.74 \pm 1.759$ days and $6.33 \pm$ 1.528 days for unsatisfactory results. This was statistically significant (p value 0.023 ).

Regarding complications, only one patient developed superficial wound infection treated by daily dressing and antibiotics for one week. Only two patients developed calcaneo-cuboid arthritis relieved by selective steroid injection. Only one patient developed tarsal tunnel syndrome and refused surgical release. Two patients developed subtalar joint pain relieved by xylocaine injection.

During the follow up period, there was a single case of superficial wound infection appeared one weeks after fixation. The patient was treated by repeated dressings and parenteral broad-spectrum antibiotics. The infection was eradicated after two weeks of treatment.

Two patients complained of "pain and tenderness" in their feet. After further assessment, the patients complained of tenderness at mid-foot at the site of calcaneocuboid joint. Analgesics failed to relieve pain. Selective injection of calcaneo-cuboid joint relieved the pain.

Two patients complained of pain and tenderness on the lateral or planter aspect of the foot, increased with walking and stressing the subtalar joint in inversion or eversion. Pain was relieved by injection of xylocaine into the joint.

One patient complained of medial heel pain and paresthesia in the distribution of the posterior tibial nerve. Pain is worse at night or with walking or standing. The diagnosis was based on the clinical picture and nerve conduction study.

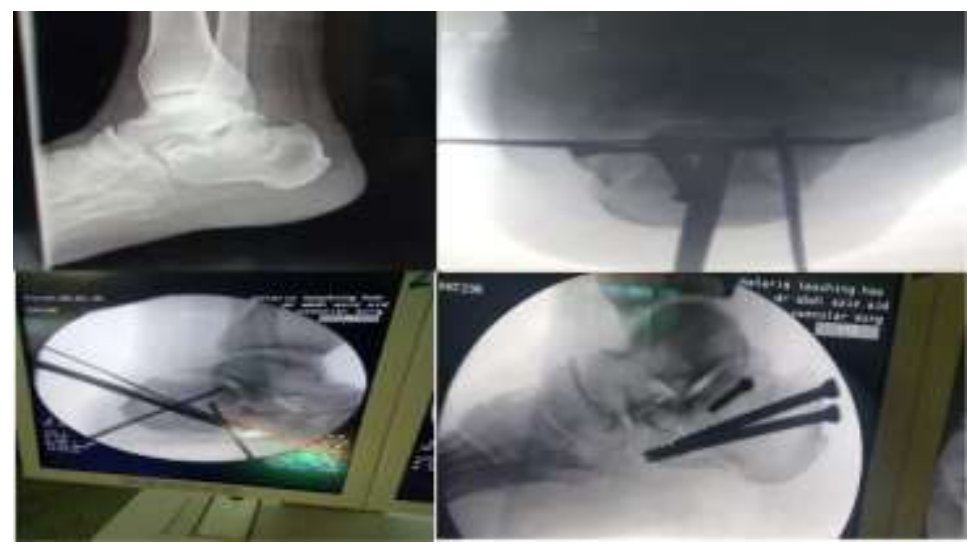

Fig. (1) preoperative, intraoperative and postoperative $\mathrm{x}$ ray ankle showing fracture calcaneaous managed by percutaneous reduction and fixation by cannulated screws.

Table (1) Classification of fractures in the current study.

\begin{tabular}{llc}
\hline Classification & \multicolumn{2}{c}{ All Fractures $(\mathbf{n}=\mathbf{2 2})$} \\
\hline Type II & 15 & $68.2 \%$ \\
Type III & 5 & $22.7 \%$ \\
Type IV & 2 & $9.1 \%$ \\
Data is expressed as percentage and frequency
\end{tabular}


Table (2) Final outcome of the studied fractures in the current study.

\begin{tabular}{llcc}
\hline Outcome & & \multicolumn{2}{c}{ All Fractures (n= 22) } \\
\hline Satisfactory & Excellent & 14 & $63.6 \%$ \\
& Good & 5 & $22.7 \%$ \\
Unsatisfactory & Fair & 2 & $9.1 \%$ \\
& Poor & 1 & $4.5 \%$ \\
\multicolumn{2}{l}{ Data is expressed as percentage and frequency } \\
\hline
\end{tabular}

Table (3) post-operative complications.

\begin{tabular}{lll}
\hline Post-operative complications & Number & Percentage \\
\hline Superficial wound infection & 1 & $4.5 \%$ \\
calcaneo-cuboid arthritis & 2 & $9.1 \%$ \\
tarsal tunnel syndrome & 1 & $4.5 \%$ \\
Subtalar joint pain & 2 & $9.1 \%$ \\
\hline
\end{tabular}

\section{Discussion}

Calcaneal fractures have long been recognized as a source of significant disability and remain one of the most difficult articular fractures to treat [2]

In this study, the mean age was $36.45 \pm 11.213$ (range from 19 to 59) years old, 42 years (range from 2276) in Rammelt S. [9] study, 36 years (range from 18 54) in Gamal O. [10] study and 49 years (range from 1773 years) in Vasukutty N. [11] study. There was no significant relationship between age and the final results of this study and also no significant relationship encountered in Rammelt S. [9] study nor Gamal O. [10] study neither Vasukutty N. study [11].

Of the 20 patients in this study was 18 patients (90\%) were males and 2 females (10\%). In Gamal O. study [10], of the 57 patients $40(70.2 \%)$ were males and $17(29.8 \%)$ were females. There was no significant relationship between gender and the final results of these studies. Male patients are much more dominant than female patients in both studies, may be due to nature of the work.

Of the 20 patients in this study, 13 patients $(65 \%)$ were broken on the right side and $5(25 \%)$ patients on the left side. There were 2 patients $(10 \%)$ of bilateral involvement. In Abdelgaid S. study [12], the right foot was injured in 16 patients, the left foot in 18 and both feet in 13 patients. There was no significant relationship between side of injury and the final results of these studies.

Of the 20 patients in this study, 18 patients $(90 \%)$ were due to fall from height and landing on the heel. The other mechanism was road traffic accident in 2 patients (10\%). In Abdelgaid S. study [12], fall from height accounted for $68.5 \%$ (32 patents). Work related injuries comprised $21 \%$ (10 patients), motor-vehicle accidents $6 \%$ (3 patents) and direct impact injuries $4.5 \%$ (2 patents). In Rammelt S. [9] study, the predominant injury mechanism was a fall from a height in $48 \%$ (16 of 33) followed by motor vehicle accidents in $36 \%$ (12 of 33). In Vasukutty N. [11] study, the mechanism of injury was fall from height in $97 \%$ and a road traffic accident in 3\% of cases. In Gamal O. study [10], of the 64 fractures 58 $(90.6 \%)$ resulted from fall from height and $6(9.4 \%)$ from road traffic injury. There was no significant relationship between mechanism of injury and the final results of these studies. Fall from height is the dominant mechanism of injury in all these studies.

The mean time from injury to operation in satisfactory results was $4.74 \pm 1.759$ days and $6.33 \pm$ 1.528 days for unsatisfactory results in this study and 7.2 days (range from 1 - 21 days) in Gamal O. study [10]. There was statistically significant relationship between time from injury to operation and the final results of these studies. (P value 0.023).

In this study regarding to associate orthopedic injuries, Only 3 patients (15\%) had associated injuries which included: fracture tibial shaft in 1 patient $(5 \%)$, lumbar spine fracture in 1 patient $(5 \%)$ and fracture femur in 1 patient (5\%). In Abdelgaid S. study [12], $25.5 \%$ of the patients (12 fractures) presented with other injuries. In Gamal O. study [10], seven of the patients $(12 \%)$ who had fallen from height also had wedge fracture of the dorso-lumber spine without any neurological manifestations.

In this study, $15(68.2 \%)$ calcaneal fractures were Type II, $5(22.7 \%)$ calcaneal fractures were Type III and $2(9.09 \%)$ calcaneal fractures were type IV. In Rammelt S. [9] study, twenty-eight (46) \% of the 61 patients had Type IIC fractures with the posterior facet being displaced as a whole. In 33(54) \% patients were Types IIA and IIB fractures. In Gamal O. study [10], 33 (51.5) $\%$ fractures were type II and $31(48.5) \%$ fractures were type III.

In this study, the mean pre-operative Böhler's angle was $14.08 \pm 2.4390$ (range $9-17$ ) and the mean postoperative Böhler's angle was $26.15 \pm 4.187$ (range 18 33 ). The result was found to be highly significant ( $p$ value $=<0.001$ ) using paired-samples T test. This means improvement of Böhler's angle after fixation achieved in 14 fractures $(63.6 \%)$ and not achieved in 8 fractures (36.4\%). In Abdelgaid S. study [12] The average preoperative Böhler's angle was 14 (9-17), compared with an average of $28(18-20)$ on postoperative evaluation. Preoperative Böhler's angle was reversed in 3 patients. In Rammelt S. [9] study, Böhler's angle improved from 12(range 0-22) preoperatively to 
25(range 16-35) postoperatively compared to 28 (range, 22-34) on the uninjured side. In Gamal O. study [10], the mean Bohler's angle improved from $6^{\circ}$ preoperatively to $26^{\circ}$ postoperatively. In Gamal O. study [10], the mean Bohler's angle was 28.18 (range 20-35) post-operatively compared with 16.4 (range 10-30) pre-operatively. All these studies showed improvement of Bohler's angle to pre-operative values after fixation.

In this study, the mean pre-operative Gissane angle was $151.14 \pm 14.264$ and the mean post-operative Gissane angle was $136.32 \pm 15.255$. The result was found to be highly significant using paired-samples $\mathrm{T}$ test (p-value $=<0.001$ ). This means improvement of Gissane angle after fixation in 12 patients $(52.2 \%)$ and not achieved in 10 patients (47.8\%). In Abdelgaid S. study [12], The average preoperative Gissane angle was 113 (range 110-155), compared with an average of 118 (range 100-138) on postoperative evaluation. In Gamal O. study [10], the mean Gissane angle was 132.27 (range 120-140) post-operatively compared with 138.64 (range 100-160) pre-operatively. All these studies showed improvement of Gissane angle to pre-operative values.

In this study, the mean pre-operative Sarrafian angle was $40.5 \pm 7.769$ and the mean post-operative Sarrafian angle was $55.32 \pm 8.555$. The result was found to be highly significant using paired-samples $\mathrm{T}$ test $(\mathrm{P}$-value $<$ 0.001).

This means improvement of Sarrafian angle after fixation in 9 patients $(43.5 \%)$ and not achieved in 13 patients (56.5\%). In Abdelgaid S. study [12], The mean preoperative Sarrafian angle was 27, with a range of (1450), with mean postoperative angle 59 (range 44-74). All these studies showed improvement of Sarrafian angle to pre-operative values after fixation.

In this study, regarding complications, only one patient developed superficial wound infection treated by daily dressing and antibiotics for one week. Only two patients developed calcaneo-cuboid arthritis relieved by selective steroid injection. Only one patient developed tarsal tunnel syndrome refused release. Two patients developed subtalar joint pain relieved by xylocaine injection. In Abdelgaid S. study [12], Secondary collapse of fragments was seen in three cases. Peroneal tendon subluxation was seen in one case. Intra-articular screw placement was seen in one case. No patients developed wound complications or infections. In Rammelt S. [9] study, no patients were observed with postoperative wound edge necrosis, hematoma, or infection. No postoperative complications related to surgery were seen, most notably compartment syndrome or any other complications attributable to subtalar arthroscopy such as fluid extravasation. In Vasukutty N. [11] study, There was one patient with wound breakdown, which healed with non-operative measures. There was one case where the screw was prominent in the subtalar joint (necessitating a repeat procedure) and one patient had sural nerve injury. There were 12 patients with symptomatic subtalar joint arthritis. Four of these had subtalar arthrodesis. In Gamal O. study [10], six patients (9.4\%) developed superficial pin tract infection that responded to local care and parenteral antibiotics therapy and completely resolved with Kirschner wire removal. Only one patient $(1.56 \%)$ developed widening of the heel. These studies revealed less wound complications with percutaneous techniques.

Regarding weight bearing, in this study, eighteen patients $(90 \%)$ started weight bearing at 12 weeks while two patients $(10 \%)$ started weight bearing at 16 weeks. In Rammelt S. [9] study, Patients returned to work at an average of 11 week. In Vasukutty N. [11] study, The mean time for return to previous vocation was 5.5 months. In Gamal O. study [10], full weight bearing was achieved within 13 (range 12-16) weeks post-operative.

The mean time to return to full activity was 4.23 (range 3-6) months. This proved that percutaneous screw fixation allow early full weight bearing and return to work.

The functional outcome in this study was evaluated using The American Orthopaedic Foot and Ankle Score (AOFAS) system and there were 14 cases $(63.6 \%)$ excellent, 5 cases $(22.7 \%)$ good , two cases $(9.1 \%)$ fair results and one case $(4.5 \%)$ poor. In Abdelgaid S. study [12], $38.3 \%$ of all cases (22 cases) had excellent results, $41 \%$ good (25 cases), fair results in $15 \%$ (9 cases), and poor results in $5 \%$ (4 cases). The overall satisfactory results (excellent and good) were $79.3 \%$. In Rammelt S. (9) study, the AOFAS mean ankle-hindfoot scale in the 24 patients was 92 . In Gamal O. study [10], the clinical results using the Maryland foot score revealed 52(81\%) with satisfactory (27 excellent and 25 good) and 12 (19\%) with unsatisfactory (10 fair and 2 poor).

\section{References}

[1] T.Tsubone, N.Toba, U.Tomoki, D.Arakawa, Prediction of fracture lines of the calcaneus using a three-dimensional finite element model. Journal of Orthopaedic Research®.vol. 37,pp. 483-489,2019.

[2] Z.Yue, Y.Tang, Z.Hu, W.Zheng, Sanders type IIIAB calcaneal fracture without broken lateral wall: A case report and review of literature. Medicine,pp. 97,2018.

[3] B.Wang, X.Zhang, Y.Huo, W.Liu, a. Calcaneal lengthening for partial traumatic loss of the calcaneus. Injury.vol. 50,pp. 796-803,2019.

[4] M.Galluzzo, , F.Greco, , M.Pietragalla, A.De Renzis, Calcaneal fractures: radiological and CT evaluation and classification systems. Acta bio-medica: Atenei Parmensis,pp. 89, 138, 2018.

[5] Liu, G. T., Vanpelt, M. D., Lalli, T., Raspovic, K. M. \& Wukich, D. K. Surgical Management of Displaced Intra-Articular Calcaneal Fractures: What Matters Most? Clinics in podiatric medicine and surgery.vol. 36,pp. 173-184, 2019.

[6] R.Buzzi, N.Sermi, F.Soviero, , Bianco Displaced intra-articular fractures of the calcaneus: ORIF through an extended lateral approach. Injury.vol. 50,pp. S2-S7, 2019.

[7] R. L.Muir, , R.Forrester, H.Sharma, Fine Wire Circular Fixation for Displaced Intra-Articular Calcaneal Fractures: A Systematic Review. The Journal of Foot and Ankle Surgery, 2019. 
[8] F.Gasparetto, G.Collo, G.Pisanu, D.Villella, Posterior ankle and subtalar arthroscopy: indications, technique, and results. Current reviews in musculoskeletal medicine.vol. 5,pp. 164-170, 2012.

[9] S.Rammelt, M.Amlang, S.Barthel, J.Gavlik, Percutaneous treatment of less severe intraarticular calcaneal fractures. Clinical Orthopaedics and Related Research®.vol. 468,pp. 983-990, 2010.

[10] O.Gamal, A.Shams, A. E.Semaya, A protocol for percutaneous transarticular fixation of sanders type II and III calcaneal fractures with or without an added mini-open approach. The Journal of Foot and Ankle Surgery.vol. 55,pp. 1202-1209, 2016.

[11] N.Vasukutty, , V.Kumar, M.Diab, W.Moussa, Operative treatment of calcaneal fractures: improved outcomes and low complications rates with a strict management protocol. The Annals of The Royal College of Surgeons of England. 99,pp. 275-279, 2017.

[12] S. M. Abdelgaid, Closed reduction and percutaneous cannulated screws fixation of displaced intra-articular calcaneus fractures. Foot and Ankle Surgery.vol. 18,pp. 164-179, 2012. 